New Zealand Pew Survey 'Spring 2021' Covid-related Results

Charles Crothers

\begin{abstract}
NZ results relating to Covid of a 'Spring' Pew Research center survey are presented. NZers tend to be strong supporters of NZ's handling of the Covid outbreak.
\end{abstract}

Key Words New Zealand, Pew, Survey, Covid

In a series of reports and accompanying data displays the Pew Research Center [https://www.pewresearch.org/] interestingly released the results of a survey of $x$ countries, which for the first time included NZ. The 'sample' of countries in this study is relatively small $(n=17)$. Some results stretching back several years from other survey rounds - and including more countries - are also included (see Appendix). 'Spring' is the Northern Hemisphere Spring. In addition, the respondent level results will be released in perhaps a year's time allowing further analyses. It may be of local import to bring the NZ results to NZ attention.

Countries covered are United States, Canada, Belgium , France, Germany, Greece, Italy, Netherlands, Spain, Sweden, United Kingdom, Australia, Japan, New Zealand, Singapore, South Korea, Taiwan - 2 North American, 9 European, 2 Antipodean and 4 Asian.

The information is focused on some important issues relating to NZ (and other) societies and includes results relating to

- Covid

- Diversity

- Climate.

This research note concentrates on covid only. Questions asked are:

- Q6b. Do you think the government of ___ respects the personal freedoms of its people

- Q7c. Would you say that each of the following has done a very good, somewhat good, somewhat bad, or very bad job dealing with the coronavirus outbreak? c. (survey public) 
- Q8 As a result of the coronavirus outbreak, has your own life changed... a great deal a fair amount, not too much not at all?

- Q9. Thinking about (survey public) as a whole, do you think this country is now More united More divided than before the coronavirus outbreak?

- Q10 Thinking about restrictions on public activity in (survey public) over the course of the coronavirus outbreak, do you think there should have been More restrictions; Fewer restrictions or The restrictions were about right

- (Survey public)'s economy is... Recovering from the effects of the coronavirus outbreak in ways that show the strengths of its economic system

- Q12. How much confidence do you have in the healthcare system in (survey public) to handle a future global health emergency that might occur

- Q29. Thinking about the economic consequences of the coronavirus outbreak, have the European Union's economic relief efforts gone too far, not gone far enough or been about right? (Included in Appendix).

A very careful methodology is deployed across countries with NZ details in the table.

New Zealand pew methods

Year: $\quad 2021$

Survey: $\quad$ Global Attitudes Survey

List-assisted Random Digit Dial (RDD) probability sample of landline households ( $30 \%$ of sample) stratified by region and RDD probability sample of cell phone users ( $70 \%$ of sample). Individuals within landline

Sample design: households are selected using the youngest adult male or female method. Interviews in the cell sample are conducted with the person who answered the phone, if age 18 or older. For both landline and cell samples, up to seven phone calls are made to complete the interview with the selected respondent.

Mode: Telephone

Language: English

Fieldwork dates: March 15 - April 30, 2021

Sample size: $\quad 1,000$

Margin of error: 3.7

Representative: Adult population 18 plus (excluding residents of Chatham Islands)

Primary vendor: Gallup

Weighting Gender, age, education, region, urbanity and probability of selection of variables: respondent

Design effects: 1.46

NZers (although eclipsed by Swedes) accord their government particularly high score for respect of personal freedoms (82\%). (NZers are more skeptical that the US Government observes similar respect for US citizens). NZers (closely followed by Singaporeans) ALSO ACCORD the government as 'very good' (74\%) in its dealing with the Covid outbreak. Although similar to Taiwan few NZers see the Covid outbreak as having changed their life a great deal (12\%). Although not so high as 
Singaporeans nevertheless a very high proportion of NZers (75\%) think NZ is more united rather than more divided compared to before the outbreak. Few NZers wanted ore restrictions (11\%). A high proportion of NZers (69\%) see its recovery as showing the strengths of its economic system. Moreover, there is considerable confidence of NZers that their health system is able to handle a future global health emergency. On all of the items, NZ is at the 'good' extreme, although not always the most satisfied country response pattern.

Tables: Pew survey results 'spring' 2021

\begin{tabular}{|c|c|c|c|c|c|c|}
\hline $\begin{array}{l}\text { Q6c. Do you think the government of } \\
\text { respects the personal freedoms of its people } \\
\text { or don't you think so? }\end{array}$ & Yes & & No & & $\mathrm{DK} / \mathrm{R}$ & Total \\
\hline United States & 63 & & 35 & & 1 & 100 \\
\hline Canada & 75 & & 24 & & 1 & 100 \\
\hline Belgium & 60 & & 39 & & 0 & 100 \\
\hline France & 58 & & 41 & & 1 & 100 \\
\hline Germany & 69 & & 30 & & 1 & 100 \\
\hline Greece & 51 & & 49 & & 1 & 100 \\
\hline Italy & 62 & & 38 & & 0 & 100 \\
\hline Netherlands & 73 & & 26 & & 0 & 100 \\
\hline Spain & 60 & & 38 & & 1 & 100 \\
\hline Sweden & 84 & & 16 & & 0 & 100 \\
\hline United Kingdom & 68 & & 32 & & 0 & 100 \\
\hline Australia & 72 & & 27 & & 1 & 100 \\
\hline Japan & 57 & & 39 & & 4 & 100 \\
\hline New Zealand & 82 & & 17 & & 1 & 100 \\
\hline Singapore & 75 & & 23 & & 1 & 100 \\
\hline South Korea & 67 & & 32 & & 1 & 100 \\
\hline $\begin{array}{l}\text { Q7c. Would you say that each of the } \\
\text { following has done a very good, somewhat } \\
\text { good, somewhat bad, or very bad job } \\
\text { dealing with the coronavirus outbreak? c. } \\
\text { (survey public) Spring, } 2021\end{array}$ & $\begin{array}{l}\text { Very } \\
\text { good }\end{array}$ & $\begin{array}{l}\text { Somew } \\
\text { hat } \\
\text { good }\end{array}$ & $\begin{array}{l}\text { Some } \\
\text { what } \\
\text { bad }\end{array}$ & $\begin{array}{l}\text { Very } \\
\text { bad }\end{array}$ & $\mathrm{DK} / \mathrm{R}$ & Total \\
\hline United States & 7 & 35 & 33 & 25 & 1 & 100 \\
\hline Canada & 14 & 51 & 20 & 14 & 0 & 100 \\
\hline Belgium & 7 & 43 & 32 & 18 & 0 & 100 \\
\hline France & 5 & 42 & 31 & 22 & 0 & 100 \\
\hline Germany & 11 & 40 & 30 & 19 & 1 & 100 \\
\hline Greece & 19 & 41 & 21 & 19 & 0 & 100 \\
\hline Italy & 13 & 46 & 21 & 20 & 0 & 100 \\
\hline Netherlands & 10 & 48 & 27 & 14 & 0 & 100 \\
\hline Spain & 10 & 34 & 25 & 30 & 1 & 100 \\
\hline Sweden & 14 & 53 & 25 & 8 & 0 & 100 \\
\hline United Kingdom & 25 & 39 & 21 & 15 & 0 & 100 \\
\hline Australia & 61 & 31 & 6 & 2 & 0 & 100 \\
\hline Japan & 4 & 31 & 47 & 17 & 1 & 100 \\
\hline New Zealand & 74 & 22 & 3 & 1 & 0 & 100 \\
\hline Singapore & 71 & 26 & 2 & 1 & 0 & 100 \\
\hline South Korea & 23 & 47 & 21 & 9 & 1 & 100 \\
\hline Taiwan & 49 & 43 & 6 & 2 & 1 & 100 \\
\hline
\end{tabular}




\begin{tabular}{|c|c|c|c|c|c|c|c|}
\hline $\begin{array}{l}\text { Q8 As a result of the coronavirus } \\
\text { outbreak, has your own life changed }\end{array}$ & $\begin{array}{l}\text { a great } \\
\text { deal }\end{array}$ & $\begin{array}{l}\text { a fair } \\
\text { amount, }\end{array}$ & \multicolumn{2}{|c|}{$\begin{array}{l}\text { not too } \\
\text { much }\end{array}$} & $\begin{array}{l}\text { not at } \\
\text { all }\end{array}$ & $\mathrm{DK} / \mathrm{R}$ & \\
\hline United States & 27 & 46 & \multicolumn{2}{|c|}{25} & 2 & 0 & 100 \\
\hline Canada & 39 & 31 & \multicolumn{2}{|l|}{25} & 6 & 0 & 100 \\
\hline Belgium & 28 & 34 & \multicolumn{2}{|l|}{$\frac{25}{30}$} & 8 & 0 & 100 \\
\hline France & 35 & 26 & \multicolumn{2}{|l|}{21} & 18 & 0 & 100 \\
\hline Germany & 28 & 29 & \multicolumn{2}{|l|}{31} & 12 & 0 & 100 \\
\hline Greece & 44 & 40 & \multicolumn{2}{|l|}{12} & 4 & 0 & 100 \\
\hline Italy & 34 & 40 & \multicolumn{2}{|l|}{18} & 8 & 0 & 100 \\
\hline Netherlands & 22 & 36 & \multicolumn{2}{|l|}{31} & 11 & 0 & 100 \\
\hline Spain & 27 & 45 & \multicolumn{2}{|l|}{22} & 6 & 0 & 100 \\
\hline Sweden & 39 & 35 & \multicolumn{2}{|l|}{22} & 4 & 0 & 100 \\
\hline United Kingdom & 34 & 31 & \multicolumn{2}{|l|}{25} & 9 & 0 & 100 \\
\hline Australia & 16 & 22 & \multicolumn{2}{|l|}{43} & 19 & 0 & 100 \\
\hline New Zealand & 12 & 21 & \multicolumn{2}{|l|}{45} & 22 & 0 & 100 \\
\hline Singapore & 27 & 36 & 31 & & 6 & 0 & 100 \\
\hline South Korea & 52 & 35 & 10 & & 2 & 0 & 100 \\
\hline Taiwan & 13 & 46 & 27 & & 14 & 0 & 100 \\
\hline $\begin{array}{l}\text { Q9. Thinking about (survey public) as } \\
\text { think } \\
\text { this country is now.. than } \\
\text { before the coronavirus outbreak? }\end{array}$ & e, do you & More L & & Mo & divided & $\mathrm{Dk} / \mathrm{R}$ & \\
\hline United States & & 10 & & 88 & & 2 & 100 \\
\hline Canada & & 36 & & 61 & & 3 & 100 \\
\hline Belgium & & 30 & & 66 & & 4 & 100 \\
\hline France & & 29 & & 68 & & 3 & 100 \\
\hline Germany & & 18 & & 77 & & 5 & 100 \\
\hline Greece & & 34 & & 61 & & 5 & 100 \\
\hline Italy & & 34 & & 63 & & 3 & 100 \\
\hline Netherlands & & 15 & & 83 & & 2 & 100 \\
\hline Spain & & 21 & & 77 & & 1 & 100 \\
\hline Sweden & & 43 & & 53 & & 3 & 100 \\
\hline United Kingdom & & 42 & & 54 & & 4 & 100 \\
\hline Australia & & 59 & & 39 & & 3 & 100 \\
\hline Japan & & 29 & & 59 & & 12 & 100 \\
\hline New Zealand & & 75 & & 23 & & 3 & 100 \\
\hline Singapore & & 86 & & 12 & & 2 & 100 \\
\hline South Korea & & 34 & & 61 & & 4 & 100 \\
\hline Taiwan & & 68 & & 20 & & 12 & 100 \\
\hline
\end{tabular}

\begin{tabular}{|l|l|l|l|l|l|}
\hline $\begin{array}{l}\text { Q10 Thinking about restrictions on } \\
\text { public activity in (survey public) over } \\
\text { the course of the coronavirus outbreak, } \\
\text { do you think there should have been... }\end{array}$ & $\begin{array}{l}\text { More } \\
\text { restrictions }\end{array}$ & $\begin{array}{l}\text { Fewer } \\
\text { restrictions }\end{array}$ & $\begin{array}{l}\text { The } \\
\text { restrictions } \\
\text { were about } \\
\text { right }\end{array}$ & & \\
\hline United States & 56 & 26 & 17 & 1 & 100 \\
\hline Canada & 53 & 18 & 27 & 2 & 100 \\
\hline Belgium & 34 & 23 & 41 & 1 & 100 \\
\hline France & 40 & 24 & 34 & 3 & 100 \\
\hline Germany & 37 & 26 & 34 & 2 & 100 \\
\hline Greece & 25 & 43 & 29 & 3 & 100 \\
\hline Italy & 32 & 21 & 46 & 1 & 100 \\
\hline Netherlands & 30 & 23 & 46 & 0 & 100 \\
\hline Spain & 46 & 13 & 40 & 1 & 100 \\
\hline Sweden & 43 & 6 & 51 & 0 & 100 \\
\hline United Kingdom & 50 & 11 & 37 & 1 & 100 \\
\hline Australia & 14 & 17 & 68 & 1 & 100 \\
\hline Japan & 62 & 8 & 25 & 4 & 100 \\
\hline New Zealand & 11 & 10 & 80 & 0 & 100 \\
\hline Singapore & 21 & 22 & 57 & 0 & 100 \\
\hline South Korea & 39 & 15 & 46 & 1 & 100 \\
\hline Taiwan & 15 & 4 & 78 & 2 & 100 \\
\hline
\end{tabular}




\begin{tabular}{|c|c|c|c|c|}
\hline (Survey public)'s economy is... & $\begin{array}{l}\text { Recovering } \\
\text { from the } \\
\text { effects of the } \\
\text { coronavirus } \\
\text { outbreak in } \\
\text { ways that } \\
\text { show the } \\
\text { strengths of } \\
\text { its economic } \\
\text { system }\end{array}$ & $\begin{array}{l}\text { Failing to } \\
\text { recover from } \\
\text { the effects of } \\
\text { the } \\
\text { coronavirus } \\
\text { outbreak in } \\
\text { ways that } \\
\text { show the } \\
\text { weaknesses of } \\
\text { its economic } \\
\text { system }\end{array}$ & $\mathrm{DK} / \mathrm{R}$ & Total \\
\hline United States & 37 & 62 & 2 & 100 \\
\hline Canada & 45 & 50 & 4 & 100 \\
\hline Belgium & 37 & 58 & 5 & 100 \\
\hline France & 25 & 71 & 4 & 100 \\
\hline Germany & 46 & 47 & 6 & 100 \\
\hline Greece & 27 & 72 & 1 & 100 \\
\hline Italy & 24 & 74 & 2 & 100 \\
\hline Netherlands & 64 & 34 & 2 & 100 \\
\hline Spain & 19 & 80 & 1 & 100 \\
\hline Sweden & 76 & 22 & 3 & 100 \\
\hline United Kingdom & 51 & 43 & 7 & 100 \\
\hline Australia & 75 & 24 & 2 & 100 \\
\hline Japan & 18 & 77 & 4 & 100 \\
\hline New Zealand & 69 & 29 & 1 & 100 \\
\hline Singapore & 77 & 22 & 1 & 100 \\
\hline South Korea & 55 & 43 & 3 & 100 \\
\hline Taiwan & 58 & 36 & 7 & $100-$ \\
\hline
\end{tabular}

\begin{tabular}{|c|c|c|c|c|c|c|}
\hline $\begin{array}{l}\text { Q12. How much confidence } \\
\text { do you have in the } \\
\text { healthcare system in (survey } \\
\text { public) to handle a } \\
\text { future global health } \\
\text { emergency that might occur } \\
\text { Spring, } 2021\end{array}$ & $\begin{array}{l}\text { a great deal } \\
\text { of } \\
\text { confidence }\end{array}$ & $\begin{array}{l}\text { some } \\
\text { confidence }\end{array}$ & $\begin{array}{l}\text { not too } \\
\text { much } \\
\text { confidence }\end{array}$ & $\begin{array}{l}\text { None at } \\
\text { all }\end{array}$ & $\mathrm{Dk} / \mathrm{r}$ & Total \\
\hline United States & 11 & 44 & 36 & 9 & 0 & 100 \\
\hline Canada & 22 & 53 & 15 & 9 & 0 & 100 \\
\hline Belgium & 23 & 49 & 20 & 9 & 0 & 100 \\
\hline France & 17 & 46 & 21 & 15 & 0 & 100 \\
\hline Germany & 38 & 37 & 15 & 9 & 1 & 100 \\
\hline Greece & 23 & 35 & 23 & 19 & 0 & 100 \\
\hline Italy & 20 & 47 & 28 & 5 & 0 & 100 \\
\hline Netherlands & 24 & 51 & 18 & 7 & 1 & 100 \\
\hline Spain & 41 & 36 & 18 & 5 & 0 & 100 \\
\hline Sweden & 33 & 50 & 12 & 4 & 0 & 100 \\
\hline United Kingdom & 39 & 44 & 12 & 5 & 1 & 100 \\
\hline Australia & 40 & 46 & 10 & 4 & 0 & 100 \\
\hline Japan & 6 & 56 & 32 & 6 & 0 & 100 \\
\hline New Zealand & 28 & 50 & 16 & 5 & 0 & 100 \\
\hline Singapore & 60 & 33 & 6 & 1 & 0 & 100 \\
\hline South Korea & 26 & 53 & 17 & 3 & 0 & 100 \\
\hline Taiwan & 39 & 49 & 10 & 1 & 1 & 100 \\
\hline
\end{tabular}

\section{Appendix: Time Series}

\begin{tabular}{|l|l|l|l|l|}
\hline $\begin{array}{l}\text { Q6b. Do you think the government of US respect } \\
\text { the personal freedoms of its people }\end{array}$ & $\begin{array}{l}\text { Yes, } \\
\text { respects } \\
\text { personal } \\
\text { freedom }\end{array}$ & $\begin{array}{l}\text { No, does } \\
\text { not } \\
\text { respect }\end{array}$ & Dk/r & Total \\
\hline Canada 2021 & 60 & 37 & 2 & 100 \\
\hline Spring, 2018 & 38 & 59 & 4 & 100 \\
\hline Spring, 2017 & 45 & 49 & 6 & 100 \\
\hline
\end{tabular}




\begin{tabular}{|c|c|c|c|c|}
\hline Spring, 2016 & 57 & 37 & 6 & 100 \\
\hline Spring, 2015 & 54 & 39 & 7 & 100 \\
\hline Spring, 2013 & 75 & 20 & 5 & 100 \\
\hline Belgium Spring, 2021 & 50 & 45 & 5 & 100 \\
\hline France Spring, 2021 & 61 & 35 & 4 & 100 \\
\hline Spring, 2018 & 40 & 57 & 3 & 100 \\
\hline Spring, 2017 & 49 & 49 & 1 & 100 \\
\hline Spring, 2016 & 44 & 51 & 4 & 100 \\
\hline Spring, 2015 & 52 & 47 & 0 & 100 \\
\hline Spring, 2014 & 69 & 30 & 0 & 100 \\
\hline Spring, 2013 & 80 & 20 & 0 & 100 \\
\hline Spring, 2008 & 65 & 35 & 0 & 100 \\
\hline Germany Spring, 2021 & 62 & 31 & 7 & 100 \\
\hline Spring, 2018 & 35 & 60 & 5 & 100 \\
\hline Spring, 2017 & 50 & 45 & 5 & 100 \\
\hline Spring, 2016 & 53 & 42 & 5 & 100 \\
\hline Spring, 2015 & 43 & 53 & 4 & 100 \\
\hline Spring, 2014 & 58 & 38 & 4 & 100 \\
\hline Spring, 2013 & 81 & 16 & 3 & 100 \\
\hline Spring, 2008 & 70 & 26 & 4 & 100 \\
\hline Greece Spring, 2021 & 53 & 43 & 4 & 100 \\
\hline Spring, 2018 & 44 & 51 & 4 & 100 \\
\hline Spring, 2017 & 46 & 48 & 6 & 100 \\
\hline Spring, 2016 & 48 & 46 & 6 & 100 \\
\hline Spring, 2014 & 43 & 53 & 4 & 100 \\
\hline Spring, 2013 & 58 & 36 & 5 & 100 \\
\hline Italy Spring, 2021 & 64 & 33 & 3 & 100 \\
\hline Spring, 2018 & 53 & 31 & 16 & 100 \\
\hline Spring, 2017 & 65 & 25 & 11 & 100 \\
\hline Spring, 2016 & 75 & 17 & 8 & 100 \\
\hline Spring, 2015 & 71 & 22 & 7 & 100 \\
\hline Spring, 2014 & 75 & 18 & 7 & 100 \\
\hline Spring, 2013 & 82 & 11 & 8 & 100 \\
\hline Netherlands Spring, 2021 & 57 & 41 & 3 & 100 \\
\hline Spring, 2018 & 38 & 59 & 4 & 100 \\
\hline Spring, 2017 & 55 & 42 & 3 & 100 \\
\hline Spring, 2016 & 54 & 41 & 5 & 100 \\
\hline 2021 & 60 & 37 & 2 & 100 \\
\hline Spain Spring, 2021 & 60 & 37 & 2 & 100 \\
\hline Spring, 2018 & 31 & 66 & 2 & 100 \\
\hline Spring, 2017 & 38 & 55 & 7 & 100 \\
\hline Spring, 2016 & 48 & 43 & 9 & 100 \\
\hline Spring, 2015 & 50 & 46 & 5 & 100 \\
\hline Spring, 2014 & 57 & 37 & 6 & 100 \\
\hline Spring, 2013 & 69 & 26 & 5 & 100 \\
\hline Spring, 2008 & 49 & 40 & 11 & 100 \\
\hline Sweden Spring, 2021 & 61 & 37 & 2 & 100 \\
\hline Spring, 2018 & 36 & 60 & 4 & 100 \\
\hline Spring, 2017 & 43 & 52 & 5 & 100 \\
\hline Spring, 2016 & 47 & 51 & 2 & 100 \\
\hline United Kingdom Spring, 2021 & 58 & 37 & 5 & 100 \\
\hline Spring, 2018 & 43 & 52 & 5 & 100 \\
\hline Spring, 2017 & 53 & 40 & 7 & 100 \\
\hline Spring, 2016 & 56 & 36 & 8 & 100 \\
\hline Spring, 2015 & 57 & 35 & 8 & 100 \\
\hline Spring, 2014 & 65 & 28 & 7 & 100 \\
\hline Spring, 2013 & 75 & 18 & 7 & 100 \\
\hline Spring, 2008 & 69 & 24 & 7 & 100 \\
\hline Australia Spring, 2021 & 50 & 48 & 2 & 100 \\
\hline Spring, 2018 & 45 & 51 & 5 & 100 \\
\hline Spring, 2017 & 54 & 42 & 4 & 100 \\
\hline Spring, 2016 & 59 & 36 & 6 & 100 \\
\hline Spring, 2015 & 58 & 34 & 7 & 100 \\
\hline
\end{tabular}




\begin{tabular}{|c|c|c|c|c|c|c|}
\hline Spring, 2013 & & 79 & 16 & & 6 & 100 \\
\hline Spring, 2008 & & 72 & 23 & & 5 & 100 \\
\hline Japan Spring, 2021 & & 59 & 31 & & 10 & 100 \\
\hline Spring, 2018 & & 66 & 28 & & 6 & 100 \\
\hline Spring, 2017 & & 69 & 24 & & 6 & 100 \\
\hline Spring, 2016 & & 76 & 17 & & 7 & 100 \\
\hline Spring, 2015 & & 76 & 17 & & 7 & 100 \\
\hline Spring, 2014 & & 84 & 10 & & 6 & 100 \\
\hline Spring, 2013 & & 85 & 9 & & 6 & 100 \\
\hline Spring, 2008 & & 80 & 17 & & 3 & 100 \\
\hline New Zealand Spring, 2021 & & 49 & 48 & & 3 & 100 \\
\hline Singapore Spring, 2021 & & 58 & 39 & & 3 & 100 \\
\hline Spain Spring, 2021 & & 76 & 22 & & 2 & 10 \\
\hline Spain Spring, 2021 & & 60 & 37 & & 2 & 100 \\
\hline Spring, 2018 & & 31 & 66 & & 2 & 100 \\
\hline Spring, 2017 & & 38 & 55 & & 7 & 100 \\
\hline Spring, 2016 & & 48 & 43 & & 9 & 100 \\
\hline Spring, 2015 & & 50 & 46 & & 5 & 100 \\
\hline Spring, 2014 & & 57 & 37 & & 6 & 100 \\
\hline Spring, 2013 & & 69 & 26 & & 5 & 100 \\
\hline Spring, 2008 & & 49 & 40 & & 11 & 100 \\
\hline Sweden Spring, 2021 & & 61 & 37 & & 2 & 100 \\
\hline Spring, 2018 & & 36 & 60 & & 4 & 100 \\
\hline Spring, 2017 & & 43 & 52 & & 5 & 100 \\
\hline Spring, 2016 & & 47 & 51 & & 2 & 100 \\
\hline United Kingdom Spring, 2021 & & 58 & 37 & & 5 & 100 \\
\hline Spring, 2018 & & 43 & 52 & & 5 & 100 \\
\hline Spring, 2017 & & 53 & 40 & & 7 & 100 \\
\hline Spring, 2016 & & 56 & 36 & & 8 & 100 \\
\hline Spring, 2015 & & 57 & 35 & & 8 & 100 \\
\hline Spring, 2014 & & 65 & 28 & & 7 & 100 \\
\hline Spring, 2013 & & 75 & 18 & & 7 & 100 \\
\hline Spring, 2008 & & 69 & 24 & & 7 & 100 \\
\hline Australia Spring, 2021 & & 50 & 48 & & 2 & 100 \\
\hline Spring, 2018 & & 45 & 51 & & 5 & 100 \\
\hline Spring, 2017 & & 54 & 42 & & 4 & 100 \\
\hline Spring, 2016 & & 59 & 36 & & 6 & 100 \\
\hline Spring, 2015 & & 58 & 34 & & 7 & 100 \\
\hline Spring, 2013 & & 79 & 16 & & 6 & 100 \\
\hline Spring, 2008 & & 72 & 23 & & 5 & 100 \\
\hline Japan Spring, 2021 & & 59 & 31 & & 10 & 100 \\
\hline Spring, 2018 & & 66 & 28 & & 6 & 100 \\
\hline Spring, 2017 & & 69 & 24 & & 6 & 100 \\
\hline Spring, 2016 & & 76 & 17 & & 7 & 100 \\
\hline Spring, 2015 & & 76 & 17 & & 7 & 100 \\
\hline Spring, 2014 & & 84 & 10 & & 6 & 100 \\
\hline Spring, 2013 & & 85 & 9 & & 6 & 100 \\
\hline Spring, 2008 & & 80 & 17 & & 3 & 100 \\
\hline New Zealand Spring, 2021 & & 49 & 48 & & 3 & 100 \\
\hline Singapore Spring, 2021 & & 58 & 39 & & 3 & 100 \\
\hline Spain Spring, 2021 & & 76 & 22 & & 2 & 100 \\
\hline South Korea Spring, 2021 & & 76 & 22 & & 2 & 100 \\
\hline Spring, 2018 & & 85 & 13 & & 1 & 100 \\
\hline Spring, 2017 & & 84 & 13 & & 3 & 100 \\
\hline Spring, 2015 & & 83 & 15 & & 2 & 100 \\
\hline Spring, 2014 & & 91 & 6 & & 3 & 100 \\
\hline Spring, 2013 & & 90 & 6 & & 3 & 100 \\
\hline Spring, 2008 & & 94 & 5 & & 1 & 100 \\
\hline Taiwan Spring, 2021 & & 74 & 17 & & 8 & 100 \\
\hline $\begin{array}{l}\text { Q7c. Would you say that each of the } \\
\text { following has done a very good, } \\
\text { somewhat good, }\end{array}$ & $\begin{array}{l}\text { Very } \\
\text { Good }\end{array}$ & $\begin{array}{l}\text { Somewh } \\
\text { at Good }\end{array}$ & $\begin{array}{l}\text { Somew } \\
\text { hat bad }\end{array}$ & $\begin{array}{l}\text { Very } \\
\text { bad }\end{array}$ & $\mathrm{DK} / \mathrm{R}$ & Tot \\
\hline
\end{tabular}




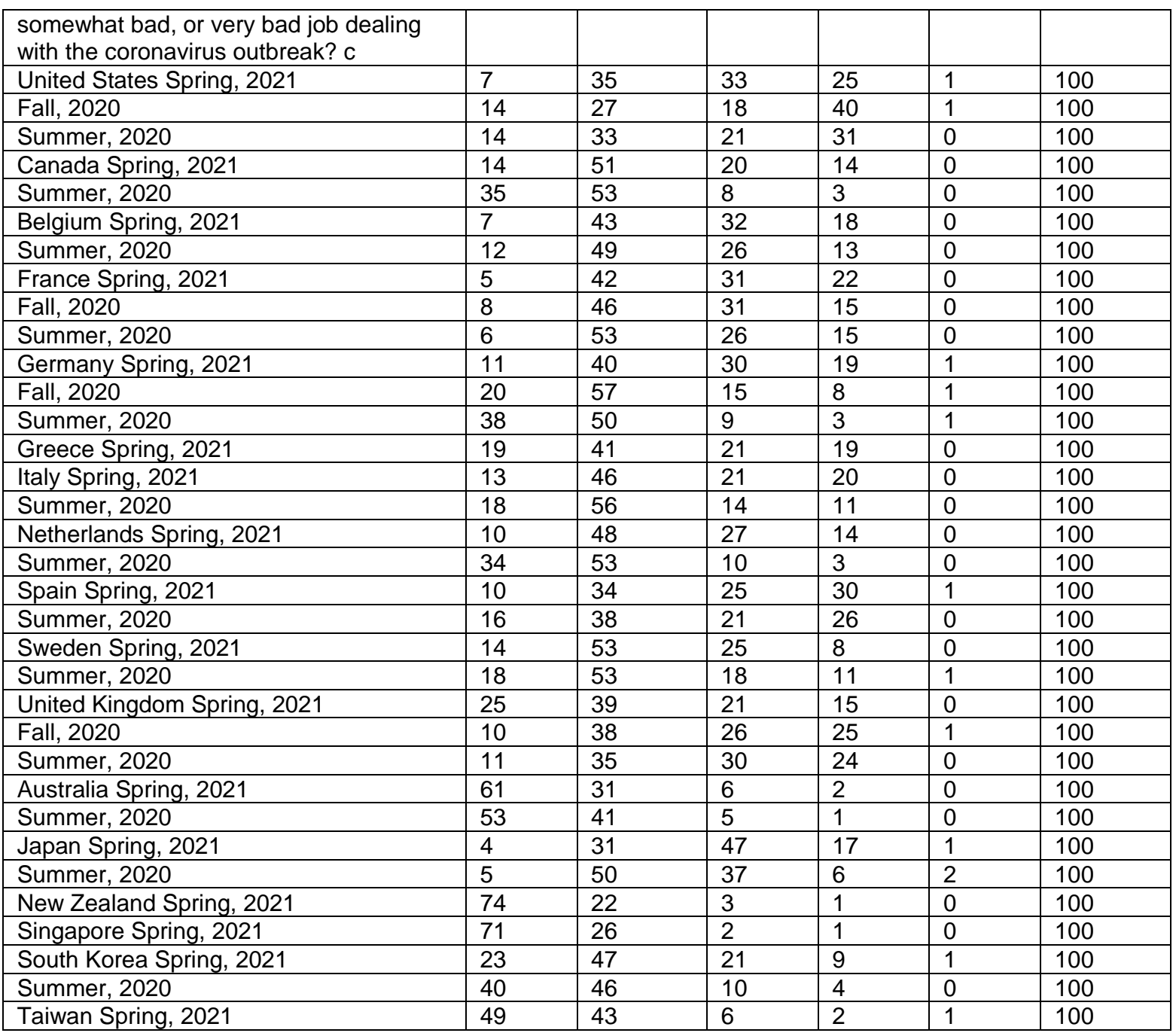

\begin{tabular}{|l|l|l|l|l|l|l|}
\hline $\begin{array}{l}\text { Q8 As a result of the coronavirus } \\
\text { outbreak, has your own life changed }\end{array}$ & $\begin{array}{l}\text {. a } \\
\text { great } \\
\text { deal }\end{array}$ & $\begin{array}{l}\text { a fair } \\
\text { amount, }\end{array}$ & $\begin{array}{l}\text { not too } \\
\text { much }\end{array}$ & $\begin{array}{l}\text { not at } \\
\text { all }\end{array}$ & DK/R & Total \\
\hline United States Spring, 2021 & 27 & 46 & 25 & 2 & 0 & 100 \\
\hline United States Fall, 2020 & 44 & 30 & 18 & 9 & 0 & 100 \\
\hline Summer, 2020 & 37 & 30 & 23 & 9 & 0 & 100 \\
\hline Canada Spring, 2021 & 39 & 31 & 25 & 6 & 0 & 100 \\
\hline Summer, 2020 & 28 & 36 & 31 & 6 & 0 & 100 \\
\hline Belgium Spring, 2021 & 28 & 34 & 30 & 8 & 0 & 100 \\
\hline Summer, 2020 & 19 & 29 & 37 & 14 & 0 & 100 \\
\hline France Spring, 2021 & 35 & 26 & 21 & 18 & 0 & 100 \\
\hline Fall, 2020 & 34 & 33 & 19 & 15 & 0 & 100 \\
\hline Summer, 2020 & 17 & 29 & 31 & 22 & 0 & 100 \\
\hline Germany Spring, 2021 & 28 & 29 & 31 & 12 & 0 & 100 \\
\hline Fall, 2020 & 21 & 26 & 37 & 15 & 0 & 100 \\
\hline Summer, 2020 & 16 & 23 & 43 & 18 & 0 & 100 \\
\hline Greece Spring, 2021 & 44 & 40 & 12 & 4 & 0 & 100 \\
\hline Italy Spring, 2021 & 34 & 40 & 18 & 8 & 0 & 100 \\
\hline Summer, 2020 & 19 & 37 & 30 & 14 & 0 & 100 \\
\hline Netherlands Spring, 2021 & 22 & 36 & 31 & 11 & 0 & 100 \\
\hline Summer, 2020 & 11 & 35 & 44 & 10 & 0 & 100 \\
\hline Spain Spring, 2021 & 27 & 45 & 22 & 6 & 0 & 100 \\
\hline Summer, 2020 & 20 & 40 & 29 & 11 & 0 & 100 \\
\hline Sweden Spring, 2021 & 39 & 35 & 22 & 4 & 0 & 100 \\
\hline Summer, 2020 & 32 & 39 & 21 & 8 & 0 & 100 \\
\hline United Kingdom Spring, 2021 & 34 & 31 & 25 & 9 & 0 & 100 \\
\hline
\end{tabular}




\begin{tabular}{|l|l|l|l|l|l|l|}
\hline Fall, 2020 & 39 & 31 & 24 & 6 & 0 & 100 \\
\hline Summer, 2020 & 32 & 34 & 27 & 6 & 0 & 100 \\
\hline Australia Spring, 2021 & 16 & 22 & 43 & 19 & 0 & 100 \\
\hline Summer, 2020 & 16 & 32 & 43 & 10 & 0 & 100 \\
\hline Japan Spring, 2021 & 21 & 48 & 24 & 6 & 0 & 100 \\
\hline Summer, 2020 & 16 & 49 & 26 & 9 & 0 & 100 \\
\hline New Zealand Spring, 2021 & $\mathbf{1 2}$ & $\mathbf{2 1}$ & $\mathbf{4 5}$ & $\mathbf{2 2}$ & $\mathbf{0}$ & $\mathbf{1 0 0}$ \\
\hline Singapore Spring, 2021 & 27 & 36 & 31 & 6 & 0 & 100 \\
\hline South Korea Spring, 2021 & 52 & 35 & 10 & 2 & 0 & 100 \\
\hline Summer, 2020 & 45 & 36 & 15 & 4 & 0 & 100 \\
\hline Taiwan Spring, 2021 & 13 & 46 & 27 & 14 & 0 & 100 \\
\hline United States Fall, 2020 & 44 & 30 & 18 & 9 & 0 & 100 \\
\hline Summer, 2020 & 37 & 30 & 23 & 9 & 0 & 100 \\
\hline
\end{tabular}

\begin{tabular}{|c|c|c|c|c|}
\hline $\begin{array}{l}\text { Q9. Thinking about (survey public) as a whole, do } \\
\text { you think } \\
\text { this country is now.. than } \\
\text { before the coronavirus outbreak? }\end{array}$ & More united & More divided & $\mathrm{Dk} / \mathrm{R}$ & Total \\
\hline United States & 10 & 88 & 2 & 100 \\
\hline Canada & 36 & 61 & 3 & 100 \\
\hline Summer, 2020 & 66 & 29 & 5 & 100 \\
\hline Belgium & 30 & 66 & 4 & 100 \\
\hline Summer, 2020 & 40 & 55 & 5 & 100 \\
\hline France & 29 & 68 & 3 & 100 \\
\hline Summer, 2020 & 39 & 49 & 12 & 100 \\
\hline Germany & 18 & 77 & 5 & 100 \\
\hline Summer, 2020 & 39 & 54 & 7 & 100 \\
\hline Greece & 34 & 61 & 5 & 100 \\
\hline Italy & 34 & 63 & 3 & 100 \\
\hline Summer, 2020 & 45 & 54 & 2 & 100 \\
\hline Netherlands & 15 & 83 & 2 & 100 \\
\hline Summer, 2020 & 44 & 53 & 3 & 100 \\
\hline Spain & 21 & 77 & 1 & 100 \\
\hline Summer, 2020 & 39 & 59 & 2 & 100 \\
\hline Sweden & 43 & 53 & 3 & 100 \\
\hline Summer, 2020 & 58 & 36 & 6 & 100 \\
\hline United Kingdom & 42 & 54 & 4 & 100 \\
\hline Summer, 2020 & 46 & 46 & 8 & 100 \\
\hline Australia & 59 & 39 & 3 & 100 \\
\hline Summer, 2020 & 54 & 40 & 6 & 100 \\
\hline Japan & 29 & 59 & 12 & 100 \\
\hline Summer, 2020 & 47 & 27 & 26 & 100 \\
\hline New Zealand & 75 & 23 & 3 & 100 \\
\hline Singapore & 86 & 12 & 2 & 100 \\
\hline South Korea & 34 & 61 & 4 & 100 \\
\hline Summer, 2020 & 56 & 36 & 8 & 100 \\
\hline Taiwan & 68 & 20 & 12 & 100 \\
\hline
\end{tabular}

\begin{tabular}{|l|l|l|l|l|l|}
\hline $\begin{array}{l}\text { Q29. Thinking about the economic } \\
\text { consequences of the [insert local term } \\
\text { for } \\
\text { coronavirus outbreak], have the } \\
\begin{array}{l}\text { European Union's economic relief } \\
\text { efforts gone } \\
\text { too far, not gone far enough or been } \\
\text { about right? }\end{array}\end{array}$ & $\begin{array}{l}\text { Gone too } \\
\text { far }\end{array}$ & $\begin{array}{l}\text { Not gone far } \\
\text { enough }\end{array}$ & $\begin{array}{l}\text { Been } \\
\text { about } \\
\text { right }\end{array}$ & Dk/R & Total \\
\hline Belgium Spring, 2021 & 10 & 43 & & & \\
\hline France Spring, 2021 & 5 & 42 & 45 & 2 & 100 \\
\hline Germany Spring, 2021 & 15 & 38 & 50 & 3 & 100 \\
\hline Greece Spring, 2021 & 3 & 60 & 43 & 4 & 100 \\
\hline Italy Spring, 2021 & 1 & 38 & 36 & 1 & 100 \\
\hline Netherlands Spring, 2021 & 7 & 30 & 60 & 1 & 100 \\
\hline
\end{tabular}


Aotearoa New Zealand Journal of Social Issues, vol. 1

Spain Spring, 2021

Sweden Spring, 2021
44 65
100

100 